\title{
Can We Understand the Clinical Biology of Gastric Cancer and Exploit it? May be, but It is a Challenge!
}

\author{
Jaffer A. Ajani, M.D. \\ Department of Gastrointestinal Medical Oncology, University of Texas M.D. Anderson Cancer Center, 1515 Holcombe Blvd,
} Houston, Texas 77030, USA

Gastric cancer remains a significant health problem worldwide, ${ }^{1}$ and yet the progress has been much slower against gastric cancer than against other cancers (colon, breast, lung, and prostate); however, the other cancers are more common in the West. Reasons for this discrepancy are many, but it is clear that if a cancer type is common in the West, it gets attention from the pharmaceutical companies and the U.S. Government. Our approach to localized gastric cancer has been typical of that for many other localized cancers; that is, we attempt a good clinical staging process and treat all defined stages similarly (empiricism). Whether we use preoperative therapy, surgery, or postoperative adjuvant therapy, one thing is certain: all patients with $>\mathrm{T} 1$ cancers when offered uniform therapy end up with diverse outcomes. Without much insight, we simply follow our patients to see who survives and who dies. We also witness unpredictable and sometime extraordinary toxicities (complications) experienced by our patients. The question is how can we possibly get out of this modus operandi? Recent molecular studies have demonstrated surprising intertumoral molecular heterogeneity that was unexpected, creating even more challenges than we thought previously. ${ }^{2}$ In gastric cancer, our limited knowledge restricts us even further, but it may be time to face that challenge.

Received July 11, 2007; accepted July 13, 2007; published online: October 12, 2007.

Commentary to Doi: 10.1245/s10434-007-9574-6

Address correspondence and reprint requests to: Jaffer A. Ajani, M.D.; E-mail: Jajani@mdanderson.org

Published by Springer Science+Business Media, LLC $\odot 2007$ The Society of Surgical Oncology, Inc.
The article by Mansour et al. ${ }^{3}$ provides some clues as to how one could possibly go about this. In this issue of the Journal, the authors from the Memorial Sloan Kettering Cancer Center relate their experience in 168 patients with curatively resected gastric cancer who received various combinations of cytotoxics preoperatively (and sometime postoperatively). The authors systematically examined the resected specimens to judge the degree of residual cancer and correlated the degree of "response" to disease-specific-survival (DSS). The authors found that the 3-year DSS was significantly different in the univariate but not in the multivariate analysis. No overall survival correlations are stated, and I am assuming that none existed. They observed that the presence of metastatic lymph nodes and perineural invasion were independently predictive of DSS in the multivariate analysis. The authors conclude that prediction and prognostication in patients with localized gastric cancer are complicated, and one may have to lean on multiple parameters (clinical and biologic) to make advances.

There are many issues worth pondering. Clearly, studies in esophageal cancer have taught us a great deal, ${ }^{4-8}$ and some studies in gastric cancer ${ }^{9-13}$ seem to show similar results. To understand this succinctly, one must recognize that all the elements of heterogeneous clinical biologic expressions are embodied in the primary cancer, and these manifest based on the level of injury one inflicts on it. Chemotherapy is less likely to lead to a manifestation of a higher level of heterogeneity compared with chemoradiation therapy (this is well known in esophageal and gastric cancers). As in the Mansour et al. study, and there are numerous examples published already; the most recent is one by 
Stahl et al. ${ }^{14}$ showing that the rate of pathologic complete response (pathCR) or even substantial response (1-50\% residual cancer) is low with chemotherapy alone compared with chemoradiation. I believe this might be one reason Monsour et al. did not see substantial correlation between patient outcome and residual cancer in the surgical specimen. The rate of pathCR is higher when one uses preoperative chemoradiation in esophageal cancers ${ }^{5-7,14}$ or gastric cancer $^{9-11}$ than when chemotherapy is used. This means that a more potent therapy seems to uncover the true nature of clinical biology of the gastric cancer better than a less potent therapy. When that happens, we begin to see the correlation between the degree of residual cancer and patient outcome. ${ }^{4,6,9-11,15}$

I agree with Mansour et al. that we should explore new ways to predict response. Positron emission tomography (PET) is one method, but there are considerable limitations of PET in gastric cancer. Nearly $45 \%$ of the cancers are not PET-avid, ${ }^{16}$ and $10 \%$ of the time, the fundus may have physiologic (false-positive) PET uptake (that may complicate interpretations). Thus the number of patients we can work with remains less than optimum and, therefore, creates another challenge. However, more specific imaging studies (evaluating proliferation, apoptosis, or oxygenation) might prove useful in this setting. In addition, studying the cancer biology might allow us to individualize therapy and follow-up.

Preoperative therapy strategy has been around for a long time, ${ }^{17-19}$ but it is only now that we are seeing some of the results of prospective evaluation, ${ }^{20,21}$ and the strategy needs considerable refinements and upgrades. Clearly, the preoperative chemoradiation approach presents itself as a worthy preoperative strategy to be compared with preoperative chemotherapy alone or with postoperative chemoradiation. The positive results from some preoperative and postoperative approaches ${ }^{20-23}$ have created an excitement as well as some confusion. The NCCN guideline (www.nccn.org) for localized gastric cancer has taken the position to accept surgery as primary therapy (to allow for postoperative adjuvant chemoradiotherapy or enrollment into the ongoing clinical trial) or preoperative approach (when the multidisciplinary infrastructure is in place). In contrast, in the countries where gastric cancer is common (Japan, Korea, Chile, Peru, China, etc.), the preoperative approach is not practiced and postoperative therapy is not a standard. We have a long way to go to unify our approaches to localized gastric carcinoma.

Finally, the residual cancer after preoperative chemoradiation can tell us a great deal about the clinical biology of gastric cancer. It can be a useful tool to communicate the prediction of patient outcome with our colleagues, patients, and families but it can also teach us a great deal about designing sophisticated trials that might prove beneficial for our patients. It is time to make noise in support of adoption of a routine multidisciplinary approach to localized gastric cancer and to explore gastric cancer biology.

\section{REFERENCES}

1. Parkin DM, Bray F, Ferlay J, Pisani P. Global cancer statistics, 2002. CA Cancer J Clin 2005; 55:74-108.

2. Sjoblom T, Jones S, Wood DL, et al. The consensus coding sequences of human breast and colorectal cancers. Science 2006; 314:268-74.

3. Mansour JC, Tang L, Shah M, et al. Does graded histologic response after neoadjuvant chemotherapy predict survival for completely resected gastric cancer? Ann Surg Oncol 2007 doi:10.1245/s10434-007-9574-6.

4. Rizk NP, Venkatraman E, Bains MS, et al. American Joint Committee on cancer staging does not accurately predict survival in patients receiving multimodality therapy for esophageal adenocarcinoma. J Clin Oncol 2007; 10:507-12.

5. Gibson M, Burtness B, Heath E, et al. Effect of neoadjuvant chemoradiotherapy on pathologic stage and survival in patients with locally advanced esophageal cancer. Proc Am Soc Clin Oncol 2004; 22:14S, (4032).

6. Chirieac LR, Swisher SG, Ajani JA, et al. Posttherapy pathologic stage predicts survival in patients with esophageal carcinoma receiving preoperative chemoradiation. Cancer 2005; 103:1347-55.

7. Berger AC, Farma J, Scott WJ, et al. Complete response to neoadjuvant chemoradiotherapy in esophageal carcinoma is associated with significantly improved survival. J Clin Oncol 2005; 23:8706-12.

8. Brucher BL, Becker K, Lordick F, et al. The clinical impact of histopathologic response assessment by residual tumor cell quantification in esophageal squamous cell carcinomas. Cancer 2006; 15:2119-27.

9. Ajani JA, Mansfield PF, Janjan N, et al. Multi-institutional trial of preoperative chemoradiotherapy in patients with potentially resectable gastric carcinoma. J Clin Oncol 2004; 22:2774-80.

10. Ajani JA, Mansfield PF, Crane $\mathrm{CH}$, et al. Paclitaxel-based chemoradiotherapy in localized gastric carcinoma: degree of pathologic response and not clinical parameters dictated patient outcome. J Clin Oncol 2005; 23:1237-44.

11. Ajani JA, Winter K, Okawara GS, et al. Phase II trial of preoperative chemoradiation in patients with localized gastric adenocarcinoma (RTOG 9904): quality of combined modality therapy and pathologic response. J Clin Oncol 2006; 24:3953-8.

12. Rohatgi PR, Mansfield PF, Crane CH, et al. Surgical pathology stage by American Joint Commission on Cancer criteria predicts patient survival after preoperative chemoradiation for localized gastric carcinoma. Cancer 2006; 107:1475-82.

13. Rohatgi PR, Mansfield PF, Crane $\mathrm{CH}$, et al. Clinical stage after preoperative chemoradiation is a better predictor of patient outcome than the baseline stage for localized gastric cancer. Cancer 2007; 110:989-95.

14. Stahl M, Walz M, Stuschke M, et al. Preoperative chemotherapy (CTX) versus preoperative chemoradiotherapy (CRTX) in locally advanced esophagogastric adenocarcino- 
mas: First results of a randomized phase III trial. 2007 ASCO Annual Meeting Proceedings. J Clin Oncol 2007.

15. Rohatgi PR, Swisher SG, Correa AM, et al. Failure patterns correlate with the proportion of residual carcinoma after preoperative chemoradiotherapy for carcinoma of the esophagus. Cancer 2005; 104:1349-55.

16. Ott K, Fink U, Becker K, et al. Prediction of response to preoperative chemotherapy in gastric carcinoma by metabolic imaging: results of a prospective trial. J Clin Oncol 2003; 21:4604-10.

17. Ajani JA, Ota DM, Jessup JM, et al. Resectable gastric carcinoma. An evaluation of preoperative and postoperative chemotherapy. Cancer 1991; 68:1501-6.

18. Leichman L, Silberman H, Leichman CG, et al. Preoperative systemic chemotherapy followed by adjuvant postoperative intraperitoneal therapy for gastric cancer: a University of Southern California pilot program. J Clin Oncol 1992; 10:1933-42.

19. Kelsen D, Karpeh M, Schwartz G, et al. Neoadjuvant therapy of high-risk gastric cancer: a phase II trial of preoperative FAMTX and postoperative intraperitoneal fluorouracil-cisplatin plus intravenous flurouracil. J Clin Oncol 1996; 14:1818-28.

20. Boige V, Pignon J, Saint-Aubert B, et al. Final results of a randomized trial comparing preoperative 5-fluorouracil $(\mathrm{F}) /$ cisplatin $(\mathrm{P})$ to surgery alone in adenocarcinoma of stomach and lower esophagus (ASLE): FNLCC ACCORD07-FFCD 9703 trial. 2007 ASCO Annual Meeting.

21. Cunningham D, Allum WH, Stenning SP, et al. Perioperative chemotherapy versus surgery alone for resectable gastroesophageal cancer. $N$ Engl J Med 2006; 355:11-20.

22. Macdonald JS, Smalley SR, Benedetti J, et al. Chemoradiotherapy after surgery compared with surgery alone for adenocarcinoma of the stomach or gastroesophageal junction. $N$ Engl J Med 2001; 345:725-30.

23. Sasako M, Yamaguchi T, Kinorshita T, et al. Randomized phase III trial comparing S-1 monotherapy versus surgery alone for stage II/III gastric cancer patients after curative D2 gastrectomy (ACTS-GC study). (Abstract No. 8). 2007 ASCO Gastrointestinal Cancers Symposium Orlando, FL: American Society of Clinical Oncology; 2007. 